\title{
COVERINGS OF PRODUCTS OF LINEAR TOPOLOGICAL SPACES
}

\author{
AARON R. TODD \\ (Received 4 March 1978; revised 26 April, 23 July 1979) \\ Communicated by A. P. Robertson
}

\begin{abstract}
An extension of the Banach-Mackey theorem is used to prove a theorem about countable families of closed balanced convex sets that cover a product of linear topological spaces. This theorem clarifies proofs that certain Baire-type properties, including the unordered Baire-like property, are preserved under products. A modification of the theorem is used to show that a property involving the bounded-absorbing sequences of DeWilde and Houet is also productive. Finally, a question is posed about balanced absorbing sets relating to products of linear Baire spaces.
\end{abstract}

1980 Mathematics subject classification (Amer. Math. Soc.): 46 A 99.

Keywords and phrases: Baire, locally convex, unordered Baire-like.

\section{Introduction}

Because translations and multiplication by nonzero scalars are homeomorphisms of a linear topological space, the following characterizes linear topological spaces that are Baire spaces: A linear topological space $E$ is a Baire space if and only if $E$ has no cover by a countable family of rare (nowhere dense) subsets. Saxon (1974b) has shown that the cover may be restricted to contain only integral multiples of a single balanced rare subset. If locally convex linear topological spaces alone are considered, and the cover contains integral multiples of a single balanced convex rare set, we obtain a characterization of barrelled spaces (those locally convex linear topological spaces all of whose closed absorbing balanced convex subsets (barrels) are neighborhoods of the origin). Other restrictions on the covers, also of an algebraic nature, result in properties intermediate to the Baire and barrelled properties (see Todd and Saxon (1973) for a detailed discussion of these properties and citations of other papers by Saxon). In particular, a locally 
convex linear topological space is unordered Baire-like if and only if it has no cover by a countable family of rare balanced convex subsets, equivalently, each cover of the space by a countable family of closed balanced convex sets contains a neighborhood of the origin. That this property strictly implies the barrelled property is easily seen from the space $(\varphi)$ of finitely nonzero sequences with its finest locally convex linear topology: $(\varphi)$ is barrelled, yet is the union of a countable family of closed proper subspaces, and thus is not unordered Baire-like. That the unordered Baire-like property is strictly implied by the Baire property for locally convex spaces was shown by Saxon (1974a). A more elementary example distinguishing these two properties is given below.

Whether or not a product of linear topological Baire spaces must be Baire seems to be an open question, although, assuming the continuum hypothesis, Oxtoby (1961) has resolved this in the negative in general topology for completely regular topological spaces. In contrast to this situation, the product of unordered Baire-like spaces is known to be unordered Baire-like (Todd and Saxon (1973), p. 32, Theorem 4.10). It is the proof of this fact that Theorem 3.1 significantly shortens and illuminates. Theorem 3.1 requires an extension of the useful BanachMackey theorem (see Horváth (1966), p. 208, Theorem 3.5.2), but before stating and proving this extension in Section 2, we give an example of an unordered Baire-like normed space which is not a Baire space.

Exampi.e. Suppose $E$ is a linear topological space whose topology $\tau$ is induced by a complete metric and is not locally convex. Furthermore, suppose that $\tau^{\circ \circ}$, the finest locally convex topology on $E$ which is weaker than $\tau$, is Hausdorff, or, equivalently, the continuous dual of $E$ separates the points of $E$. In particular, $E=l^{\frac{1}{2}}$ with the usual paranormed topology for $\tau$ satisfies these requirements, moreover $\tau^{\circ \circ}$ is induced by the $l^{1}$-norm so that $\left(E, \tau^{\circ 0}\right)$ is a normed space in this case (see Robertson (1958), p. 256).

Let $\left\{B_{n}\right\}_{n \geqslant 1}$ be a cover of $E$ by balanced convex subsets of $E$ that are $\tau^{\circ \circ}$ closed. Now $\left\{B_{n}\right\}$ is a countable cover of $E$ by $\tau$-closed sets as $\tau^{\circ \circ}$ is coarser than $\tau$. Since $(E, \tau)$ is a complete metrizable space, some $B_{n}$ is a $\tau$-neighborhood of some point. Since $B_{n}$ is balanced and convex, it contains $\frac{1}{2} B_{n}-\frac{1}{2} B_{n}$, a $\tau$-neighborhood of the origin, and so $B_{n}$ is also a $\tau^{\circ 0}$-neighborhood of the origin. Therefore, under $\tau^{\circ 0}, E$ is an unordered Baire-like space.

Finally, consider the inclusion $f:(E, \tau) \hookrightarrow\left(E, \tau^{\circ \circ}\right)$, which is linear, surjective, injective and continuous. Since $(E, \tau)$ is a complete metrizable space, $f$ is open if $\left(E, \tau^{\circ \circ}\right)$ is a Baire space (see Kelley and Namioka (1963), p. 99, Theorem 11.4). As $\tau$ is not locally convex, $f$ is not open, and so under $\tau^{\circ 0}, E$ is not a Baire space.

In the sequel, we shall conveniently shorten certain phrases, for example, 'locally convex linear topological space' becomes 'convex space' and 'neighborhood of the origin of E' becomes 'neighborhood in $E$ '. 


\section{The Banach-Mackey theorem}

The following is an extension of the Banach-Mackey theorem, which, in one of its forms, states that a barrel of a linear topological space absorbs each closed balanced convex subset that is bounded and complete. In the following, the usual Grothendieck notation is used: For $C$ a balanced convex subset of a linear space $E$, let $E_{C}$ be the linear subspace $\bigcup_{n \geqslant 1} n C$ of $E$ equipped with the seminorm induced by $C$ (refer to Horváth (1966), p. 207).

2.1 THEOREM. If a linear topological space $E$ is covered by a countable family $\mathscr{B}$ of sequentially closed balanced convex sets, then each balanced convex bounded subset $C$ of $E$ such that $E_{C}$ is unordered Baire-like is absorbed by some element of $\mathscr{B}$.

Proof. Without loss of generality, assume that $C$ is absorbing in $E$, and hence induces a seminormed topology $v$ on $E$, which, since $C$ is bounded, is finer than the original topology of $E$. Now, as $E=E_{C}$ is unordered Baire-like for the topology $v$, some element $B$ of the cover $\mathscr{B}$ is not $v$-rare. Since $\mathscr{B}$ is sequentially closed in the original topology, $B$ is closed in the finer seminormed topology $v$, and so $B$ is a $v$-neighborhood of some point. But $B$ is balanced and convex, thus $B$ is a neighborhood in $E_{C}$ and so absorbs $C$.

A common enough case in which the seminormed space $E_{C}$ of the theorem is unordered Baire-like arises when $E_{C}$ is complete and, therefore, is a Baire space. This is the case when the bounded balanced convex set $C$ is nonempty closed and complete (see, for example, Horváth (1966), p. 207, Proposition 3.5.6, but modified for non-Hausdorff spaces). In the following corollary, 'complete' may be replaced by 'compact' in which form the corollary will be used for Theorem 3.1.

2.2 Corollary. If a linear topological space $E$ is covered by a countable family $\mathscr{B}$ of closed balanced convex sets, then each closed balanced convex subset of $E$ which is complete is absorbed by some element of $\mathscr{B}$.

The following permanence property for barrelled spaces is well known, but generally proved with duality (see Horváth (1966), p. 270, Exercise 3.14.2(a)). The observation that it may be proved using a technique based on the BanachMackey theorem appears in Adasch (1970), p. 282, Remark 1. As the proof of Theorem 3.1 is a complicated variation of this technique, the proof of the following is omitted. 
2.3 COROLLARY. The product of barrelled spaces is barrelled.

As a direct corollary of Theorem 4.1 of Todd and Saxon (1973), the following requires no topology and is true without the requirement that $\mathscr{B}$ consist of closed sets; however, the present formulation is adequate for its use in Theorem 3.1.

2.4 COROLlaRY. If a linear topological space $E$ is covered by the union of two countable families, $\mathscr{B}_{1}$ and $\mathscr{B}_{2}$, of closed balanced convex sets, then either $\bigcup \mathscr{B}_{1}$ or $\mathrm{U} \mathscr{B}_{2}$ is absorbing in $E$.

Proof. Assume $x$ and $y$ are elements of $E$ which are not absorbed by $\bigcup \mathscr{B}_{1}$ and $\bigcup \mathscr{B}_{2}$, respectively, and let $C$ be the closed balanced convex hull of $\{x, y\}$. Since $C$ is the closure of a compact subset of a regular space, it is compact, and Corollary 2.1 implies that some element, say $B$, of $\mathscr{B}_{1} \cup \mathscr{B}_{2}$ absorbs $C$. Thus $B$ absorbs both $x$ and $y$, and, since $B$ is in either $\mathscr{B}_{1}$ or $\mathscr{B}_{2}$, one of $\bigcup \mathscr{B}_{1}$ and $\bigcup \mathscr{B}_{2}$ absorbs both $x$ and $y$, a contradiction.

\section{The main theorem}

The next theorem relies heavily on the discussion of Section 2, is rather technical, and is central to the proof that the arbitrary product of unordered Baire-like spaces is unordered Baire-like. The notation has been simplified by identifying the direct sum $S=\sum_{J} E_{j}$ and product $P=\prod_{J} E_{j}$ of linear spaces $E_{j}, j \in J$, with subspaces of a 'larger' product $E=\prod_{I} E_{i}$ of linear spaces $E_{i}, i \in I$, where the index set $I$ contains the index set $J$. More specifically, let $F$ be either $S$ or $P$, then $F$ is identified with the following subspace of $E$ :

$\{x \in E$ : for all $i \in I \backslash J, x(i)=0$,

and there exists $y \in F$ such that for all $j \in J, x(j)=y(j)\}$.

3.1 MaIN TheOREM. If the product $E$ of linear topological spaces $E_{i}, i \in I$, is covered by a countable family $\mathscr{B}$ of closed balanced convex subsets of $E$, then

(i) either some member of the cover $\mathscr{B}$ absorbs each element of the product $E$,

(ii) or there is a factor space $E_{i}$ such that the union of all members of the family $\mathscr{B}$ that are not absorbing in $E_{i}$ absorbs each point of the factor space $E_{i}$.

Proof. If $I$ is not infinite, make it so with the inclusion of $\{0\}$-factors. Let $\mathscr{B}_{1}=\left\{B \in \mathscr{B}\right.$ : for every finite $\left.J \subset I, B \oplus \sum_{I \backslash J} E_{i}\right\}$. By Corollary 2.4 , either $\bigcup \mathscr{B}_{1}$ or $\bigcup\left(\mathscr{B} \mid \mathscr{B}_{1}\right)$ is absorbing in $E$. Let $\left\{B_{n}\right\}_{n \geqslant 1}=\mathscr{B}_{1}$.

CLAIM. There is an increasing sequence $\left(J_{k}\right)_{k} \geqslant 0$ of finite subsets of I and a sequence 
$\left(x_{k}\right)_{k \geqslant 1}$ of elements of $E$ with $J_{k} \backslash J_{k-1}=\operatorname{supp}\left(x_{k}\right)=\left\{i \in I: x_{k}(i) \neq 0\right\}$ such that for each $B_{n}$ and integer $m$, there is an integer $k>m$ for which $x_{k}$ is not in $k B_{n}$.

OUtLINe of Proof. Start with $J_{0}=\varnothing$. By the choice of $\mathscr{B}_{1}$, the set $B_{1}$ does not contain all of $\sum\left\{E_{i}: i \in I \backslash J_{0}\right\}$, and so there is a point $x_{1}$ in $\left(\sum\left\{E_{i}: i \in I \backslash J_{0}\right\}\right) \backslash B_{1}$. Let $J_{1}=J_{0} \cup \operatorname{supp}\left(x_{1}\right)$, which is finite. As $B_{2}$ does not contain $\sum\left\{E_{i}: i \in I \backslash J_{1}\right\}$, there is a point $x_{2}$ in $\left(\sum\left\{E_{i}: i \in I \backslash J_{1}\right\}\right) \backslash 2 B_{2}$. Let $J_{2}=J_{1} \cup \operatorname{supp}\left(x_{2}\right)$. As before, there is $x_{3}$ in $\left(\sum\left\{E_{i}: i \in I \backslash J_{2}\right\}\right) \backslash 3 B_{1}$, and we let $J_{3}=J_{2} \cup \operatorname{supp}\left(x_{3}\right)$. There is $x_{4}$ in $\left(\sum\left\{E_{i}: i \in I \backslash J_{3}\right\}\right) \backslash 4 B_{2}$, and we let $J_{4}=J_{3} \cup \operatorname{supp}\left(x_{4}\right)$. Let $x_{5}$ be in

$$
\left(\sum\left\{E_{i}: i \in I \backslash J_{4}\right\}\right) \backslash 5 B_{3} \text {, and } J_{5}=J_{4} \cup \operatorname{supp}\left(x_{5}\right) \text {. }
$$

Now repeat the basic step with each of $B_{1}, B_{2}$, and $B_{3}$ in that order. In general, repeat the basic step with each of $B_{1}, B_{2}, B_{3}, \ldots, B_{n+1}$, in that order, after its first application to $B_{n+1}$.

Now as $\left\{J_{k} \mid J_{k-1}\right\}_{k} \geqslant 1$ is a disjoint family of subsets of the index set $I$, there is a member $x_{0}$ of the product $E$ defined by $x_{0}(i)=x_{k}(i)$, if $i$ is in $J_{k} \backslash J_{k-1}=\operatorname{supp}\left(x_{k}\right)$, and $x_{0}(i)=0$, if $i$ is in $I \backslash \bigcup_{m \geqslant 0} J_{m}$. Let $K$ be the closure of the compact set $\left\{x \in E: x(i)=\lambda_{i} x_{0}(i),\left|\lambda_{i}\right| \leqslant 1, i \in I\right\}$. Now $K$ is a closed balanced convex subset of $E$, which is compact and contains the sequence $\left(x_{k}\right)$. By Corollary 2.2 , some $B_{n}$ absorbs $K$; that is, for some positive integer $m$, the set $K$ is contained in $m B_{n}$. Yet, from the above claim, there is an integer $k$ larger than $m$ such that $x_{k}$ is not in $k B_{n}$, and so $x_{k}$ is not in the subset $m B_{n}$ of $k B_{n}$, a contradiction of the fact that $m B_{n}$ contains $K$. Hence the assumption that $\bigcup \mathscr{B}_{1}$ is absorbing in $E$ is contradictory, and, by Corollary 2.4, $U\left(\mathscr{B} \mid \mathscr{B}_{1}\right)$ is absorbing in $E$.

For convenience, we may suppose that each element $B$ of $\mathscr{B}$ contains $\sum_{I \backslash J} E_{i}$ for some finite subset $J$ of $I$. Let us assume that conclusion (i) of the theorem is false, so that no member of $\mathscr{B}$ is absorbing in the product $E$. For each $i$ in $I$, let $\mathscr{B}_{i}=\left\{B \in \mathscr{B}: B\right.$ is not absorbing in $\left.E_{i}\right\}$. The union of the family $\left\{\mathscr{B}_{i}\right\}_{i \in I}$ is $\mathscr{B}$, for suppose otherwise, then some element $B$ of $\mathscr{B}$ is absorbing in each $E_{i}$. There is a finite subset $J$ of $I$, such that $B$ contains $\sum_{I \backslash J} E_{i}$. But $B$ is closed and $\sum_{I \backslash J} E_{i}$ is

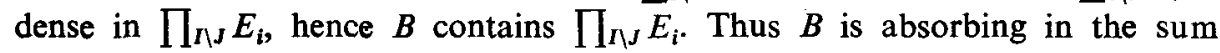
$\prod_{J} E_{j}+\prod_{r_{J} J} E_{i}$ which is $E$, in contradiction of the assumption that (i) is false. Therefore $\bigcup_{I} \mathscr{B} i=\mathscr{B}$, as claimed.

Let us assume that (ii) is also false, so that no $\bigcup \mathscr{B}_{i}$ is absorbing in $E_{i}$. Hence, for each $i$ in $I$, there is an element $x_{i}$ of $E_{i}$ such that $\bigcup \mathscr{B}_{i}$ does not absorb $x_{i}$. Define $x_{0}$ in $E$ by $x_{0}(i)=x_{i}(i)$ for each $i$ in $I$, and let $K$ be the closure of the compact set $\left\{x \in E: x(i)=\lambda_{i} x_{0}(i),\left|\lambda_{i}\right| \leqslant 1, i \in I\right\}$. The set $K$ is a closed balanced convex subset of $E$, which is compact and contains each $x_{i}, i \in I$. By Corollary 2.3, $K$ is absorbed by some member $B$ of $\mathscr{B}$. But this is contradictory as $B$ is in some $\mathscr{B}_{i}$, the point $x_{i}$ is in $K$, and $\bigcup \mathscr{B}_{i}$ does not absorb $x_{i}$. Therefore conclusions (i) and (ii) cannot both be false. 
The following is now a relatively simple corollary of the above theorem.

3.2 Corollary [Todd and Saxon (1973)]. The product of unordered Baire-like spaces is unordered Baire-like.

Proof. Let $E$ be a product of unordered Baire-like spaces $E_{i}, i \in I$, and suppose that $\mathscr{B}$ is a countable family of closed balanced convex subsets of $E$ which covers $E$. If $\mathscr{B}$ contains no member which is absorbing in $E$, then, from Theorem 3.1, there is a factor space $E_{i}$ covered by

$\mathscr{B}_{i}=\{k B: k$ is an integer, and $B$ is a member of $\mathscr{B}$ which is not absorbing in $\left.E_{i}\right\}$.

Now $\left\{B \cap E_{i}: B \in \mathscr{B}_{i}\right\}$ is a cover of $E_{i}$ by a countable family of closed balanced convex subsets of $E_{i}$. As $E_{i}$ is unordered Baire-like, there is a $B$ in $\mathscr{B}_{i}$ such that $B \cap E_{i}$ is a neighborhood in $E_{i}$, and so $B$ is absorbing in $E_{i}$. This contradicts the characterization of $\mathscr{B}_{i}$, and there must, in fact, be a member $C$ of $\mathscr{B}$ which is absorbing in $E$. Now $C$ is a barrel in $E$, which is barrelled by Corollary 2.3. Hence $C$ is a neighborhood in $E$, and therefore $E$ is unordered Baire-like.

The main theorem, as technical as it appears, puts in better focus the lemmas of Todd and Saxon (1973) leading up to the above fact and may be used in product results of other properties cited in their paper. Although the theorem is mainly directed towards this end, it and its proof have other consequences, one of which we shall now investigate.

We shall say that a family $\mathscr{B}$ of subsets of a linear topological space $E$ is bornivorous for a linear subspace $F$ of $E$ if each bounded subset of $F$ is absorbed by some member of the family $\mathscr{B}$. Thus a set $B$ is bornivorous (see Horváth (1966), p. 210, Definition 3) in a linear topological space $E$ if the singleton $\{B\}$ is a bornivorous family for $E$. A specialized example of a bornivorous family is discussed by DeWilde and Houet (1971): An increasing sequence $\left(B_{n}\right)$ of balanced convex subsets of a linear topological space $E$ is a bounded-absorbent sequence if each bounded set of $E$ is absorbed by some term $B_{n}$ of the sequence.

The proof of the main theorem is now easily modified for the following theorem in which the cover is linked to bounded sets. The one significant difference in the proof is that the Banach-Mackey theorem is no longer needed as the absorption of bounded sets forms part of the hypotheses.

3.3 THEOREM. If the product $E$ of linear topological spaces $E_{i}, i \in I$, has a countable bornivorous family $\mathscr{B}$ of closed balanced convex subsets of $E$, then

(i) either some member of the family $\mathscr{B}$ absorbs each bounded set of the product $E$,

(ii) or there is a factor space $E_{i}$ such that the family of all members of $\mathscr{B}$ that are not bornivorous in $E_{i}$ forms a bornivorous family for $E_{i}$. 
DeWilde and Houet (1971), p. 259, Corollary 2.d, state conditions that give a convex space $E$ the following property: If $\left(C_{n}\right)$ is a bounded-absorbent sequence of closed sets in $E$, then some term $C_{n}$ is a neighborhood in $E$. In particular, dense quasi-barrelled subspaces of a convex Baire space have this property. We may now use the above theorem to show that this property is productive.

3.4 COROLlary. If $E$ is the product of linear topological spaces $E_{i}, i \in I$, each of which has the property that each bounded-absorbent sequence of closed sets contains a neighborhood, then $E$ has the same property.

Proof. Let $\left(C_{n}\right)$ be a bounded-absorbent sequence of closed sets of $E=\prod_{I} E_{i}$. By Theorem 3.3, some $C_{n}$ is bornivorous in $E$, or, since $\left(C_{n}\right)$ is increasing, there is an $i$ in $I$ such that no $C_{n}$ is bornivorous in $E_{i}$. As the latter contradicts the required property of $E_{i}$, we may suppose that $C_{n}$ is bornivorous in $E$. From the proof of Theorem 3.1, there is a finite subset $J$ of $I$ with $\prod_{I \backslash J} E_{i} \subset C_{n}$. Moreover, for each $j$ in $J$ there is a neighborhood $U_{j}$ in $E_{j}$ such that $U_{j} \subset(1 / N) C_{n}$, where $N=|J|+1$, and so, as $C_{n}$ is balanced and convex,

$$
\left(\prod_{J} U_{J}\right) \times \prod_{I \backslash J} E_{i}=\sum_{J} U_{j}+\prod_{I \backslash J} E_{i} \subset C_{n} .
$$

Therefore $C_{n}$ is a neighborhood in $E$.

Products of certain linear topological spaces have no cover by a countable family of closed balanced convex sets unless one of the sets contains the whole space; such a cover will be called a trivial cover.

3.5 COROLlary. If $E$ is the product of linear topological spaces $E_{i}, i \in I$, each of which has only trivial covers by countable families of closed balanced convex sets, then any subspace $F$ of $E$ which contains every point of $E$ whose coordinates are countably nonzero has only trivial covers by countable families of closed balanced convex sets.

Proof. Suppose $\mathscr{B}=\left\{B_{n}\right\}_{n \geqslant 1}$ is a family of closed balanced convex subsets of $E$ which covers $F$. It is readily seen (Todd and Saxon (1973), p. 31, Lemma 4.9) that $\mathscr{B}$ covers $E$. As no factor $E_{i}$ may be covered by

$\mathscr{B}_{i}=\{k B: k$ is an integer, and $B$ is a member of $\mathscr{B}$ which is not absorbing in $E_{i}$,

Theorem 3.1 implies that there is an element $B$ of $\mathscr{B}$ which is absorbing in $E$. Hence $\{k B\}_{k \geqslant 1}$ is a cover of $E$, and, by hypothesis, each $E_{i}$ is contained in some $k B$. As each $E_{i}$ is a linear space, $B$ contains each $E_{i}$. Let $x_{j}$ be an element of $E_{j}$ for each $j$ in a finite nonempty subset $J$ of $I$. With $n=|J| \neq 0$, let

$$
x=\sum x_{j}=\sum_{J} n x_{j} / n .
$$


By convexity, $x$ is an element of $B$. Since $x$ is an arbitrary element of the sum $\sum_{\mathrm{I}} E_{i}$, the closed set $B$ contains $\prod_{l} E_{i}$, and the cover $\mathscr{B}$ is trivial.

The following example uses the above corollary.

3.6 ExAMPLE. Suppose $E_{i}, i \in I$, are linear Baire spaces each of which has no nontrivial convex open subset. The $E_{i}, i \in I$, satisfy the requirements of the corollary. In particular, if each $E_{i}$ is the linear topological space of Lebesgue measurable functions on $[0,1]$ with convergence in measure, then each $E_{i}$ is a complete linear metrizable space having no nontrivial convex open set (Mazur and Orlicz (1948), p. 208). Although the complete space $E=\prod_{I} E_{i}$ need not be metrizable, the result of the corollary may also be obtained for this choice of $E_{i}, i \in I$, from the fact that a product of complete metrizable spaces is Baire (Bourbaki (1948), p. 4, Exercise 7), and such subspaces $F$ of $E$, as in the corollary, are Baire if $E$ is Baire (Todd and Saxon (1973), p. 32, Theorem 4.11).

\section{Sub-seminorms and an open question}

The barrelled property of locally convex spaces is characterized by the continuity of every lower semicontinuous seminorm (Horváth (1966), p. 219, Exercise 3.6.1). We shall obtain the analogous characterization of unordered Baire-like spaces and a different form of Theorem 3.1 through an extension of the concept of seminorm. A duality result of Theorem 3.1 will be given which is equivalent to Theorem 3.1 in the context of convex spaces. This result may be compared with the duality characterization of the unordered Baire-like property obtained by Saxon (1974a).

For a balanced convex set $B$ of a linear space $E$, the gauge $p$ of $B$ is defined at each point $x$ of $E$ by

$$
p(x)= \begin{cases}\inf \Lambda, & \Lambda=\{\lambda>0: x \in \lambda B\} \neq \varnothing, \\ +\infty, & \text { otherwise }\end{cases}
$$

Since the balanced convex set $B$ is absorbing in its linear span, $F=\bigcup_{n \geqslant 1} n B$, the restriction of $p$ to $F$ is a seminorm on $F$ (see Horváth (1966), p. 94). Conversely, if $p$ is a seminorm on a linear subspace $F$ of $E$, then $p$ is the restriction to $F$ of the gauge of the balanced convex set $\{x \in F: p(x) \leqslant 1\}$. We shall call the gauge of a balanced convex subset $B$ of a linear topological space $E$, a sub-seminorm of $E$ induced by $B$. A sub-seminorm of $E$ is equivalently characterized as a non-negative extended real-valued function on $E$ that is absolutely homogeneous and satisfies the triangle inequality. It may be verified that a closed balanced convex subset $B$ of a linear topological space $E$ induces a sub-seminorm $p$ on $E$ which is lower semicontinuous and $B=\{x \in E: p(x) \leqslant 1\}$. Conversely, if $p$ is a sub-seminorm of 
a linear topological space $E$ that is lower semicontinuous, then the balanced convex set $B=\{x \in E: p(x) \leqslant 1\}$ is closed.

4.1 Proposition. A locally convex space $E$ is unordered Baire-like if and only if the following condition is satisfied: If a countable family $\mathscr{P}$ of lower semicontinuous sub-seminorms of $E$ contains, for each point $x$ of $E$, some member $p$ with $p(x)$ finite, then $\mathscr{P}$ contains a continuous seminorm.

Proof. Suppose $E$ has a cover by a countable family $\mathscr{B}$ of closed balanced convex subsets of $E$. Let $\mathscr{P}$ be the countable family of lower semicontinuous subseminorms of $E$ induced by the members of $\mathscr{B}$. For each point $x$ of $E$, there is a member $B$ of $\mathscr{B}$ containing $x$. Now the gauge $p$ of $B$ has a value less than or equal to 1 at $x$. If the condition of the proposition is satisfied, some member $q$ of $\mathscr{P}$ is a continuous seminorm of $E$. Thus $C=\{x \in E: q(x) \leqslant 1\}$ is a neighborhood in $E$, and there is a member $C^{\prime}$ of $\mathscr{B}$ which induces $q$ on $E$. Since $q$ is a continuous seminorm and $C^{\prime}$ is closed, $C^{\prime}=C$, and $C^{\prime}$ is a member of $\mathscr{B}$ that is a neighborhood in $E$. Therefore $E$ is unordered Baire-like when the condition is satisfied.

Conversely, suppose that $E$ is unordered Baire-like and $\mathscr{P}$ satisfies the hypotheses of the condition. Since $E$ is unordered Baire-like, some member of the cover of $E$ by sets of the form $k\{x \in E: p(x) \leqslant 1\}$, where $k$ is a positive integer and $p$ is an element of $\mathscr{P}$, is a neighborhood in $E$. Therefore some element of $\mathscr{P}$ is a continuous seminorm of $E$.

Using the concept of sub-seminorm, we restate Theorem 3.1 without proof.

4.2 THEOREM. If a countable family $\mathscr{P}$ of lower semicontinuous sub-seminorms of a product $E$ of linear topological spaces $E_{i}, i \in I$, contains, for each point $x$ of $E$, some member $p$ with $p(x)$ finite, then

(i) either there is a member of $\mathscr{P}$ that is a seminorm of $E$

(ii) or there is a factor space $E_{i}$ such that the family $\mathscr{P}_{i}$ of all members of $\mathscr{P}$ that are not finite valued on $E_{i}$ contains, for each point $y$ of $E_{i}$, some member $q$ such that $q(y)$ is finite.

For convex spaces, the following is equivalent to Theorem 3.1.

4.3 THEOREM. If a countable family $\mathscr{A}$ of subsets of the continuous dual $E^{\prime}$ of the product $E$ of linear topological spaces $E_{i}, i \in I$, contains, for each point $x$ of $E, a$ member which is bounded at $x$, then

(i) either there is a member of $\mathscr{A}$ which is pointwise bounded on $E$,

(ii) or there is a factor space $E_{i}$, such that the family $\mathscr{A}_{i}$ of all members of $\mathscr{A}$ which are not pointwise bounded on $E_{i}$ contains, for each point $y$ of $E_{i}$, some member $A$ which is bounded at $y$. 
Proof. For $A$ in $\mathscr{A}$, define $p_{A}$ at each point $x$ of $E$ by $p_{A}(x)=\sup \{|\langle x, f\rangle|: f \in A\}$. It is easily seen that $p_{A}$ is a non-negative extended real-valued function that is absolutely homogeneous and satisfies the triangle inequality, so that $p_{A}$ is a subseminorm of $E$. Moreover, $p_{A}$ is the pointwise supremum of continuous functions, $x \mapsto|\langle x, f\rangle|, f \in A$, so $p_{A}$ is lower semicontinuous. For a point $x$ of $E$, there is a member $A$ of $\mathscr{A}$ with $A$ bounded at $x$, hence the corresponding sub-seminorm $p_{A}$ is finite at $x$. Therefore $\mathscr{P}=\left\{p_{A}: A \in \mathscr{A}\right\}$ satisfies the condition on $\mathscr{P}$ in Theorem 4.2.

Suppose for some $A$ in $\mathscr{A}, p_{A}$ is a seminorm of $E$, then, clearly, $A$ is bounded at each point of $E$. On the other hand, if part (i) of the above is false, then let $\mathscr{P}_{i}$ and $E_{i}$ be as in result (ii) of Theorem 4.2. Evidently, $\mathscr{P}_{i}$ is $\left\{p_{A}: A \in \mathscr{A}_{i}\right\}$. Thus, for each $y$ in $E_{i}$ some element $p_{A}$ of $\mathscr{P}_{i}$ is finite at $x$, and so, in turn, $A$ of $\mathscr{A}_{i}$ is bounded at $x$.

We end the paper with an open question that may have a bearing on the problem of whether or not a product of a family of linear Baire spaces is a Baire space. The Banach-Mackey theorem may be extended in the following way: A closed absorbing set $B$ of a linear topological space $E$ absorbs each closed balanced convex compact set $C$ at some point $x$ of $B$. Question: Is there a point $x$ of $B$ at which $B$ absorbs each closed balanced convex compact subset $C$ of $E$ ?

ADDED IN PROOF: For additional examples of unordered Baire-like spaces that are not Baire, see: P. Dierolf, S. Dierolf and L. Drewnowski (1978), Colloq. Math. 39, 109-116.

\section{References}

N. Adasch (1970), 'Topologische Produkte gewisser topologisher Vektorräume', Math. Ann. 189, 280-284.

N. Bourbaki (1948), Topologie générale, Chapter 9 (Act. Sci. Ind. No. 1045, Hermann, Paris).

M. DeWilde and C. Houet (1971), 'On increasing sequences of absolutely convex sets in locally convex spaces', Math. Ann. 192, 257-261.

J. Horváth (1966), Topological vector spaces and distributions, Vol. 1 (Addison-Wesley, Reading, Mass.).

J. L. Kelley, I. Namioka et al. (1963), Linear topological spaces (D. Van Nostrand, Princeton, New Jersey).

S. Mazur and W. Orlicz (1948), 'Sur les espaces métriques linéaires I', Studia Math. 10, 184-208.

J. C. Oxtoby (1961), 'Cartesian products of Baire spaces', Fund. Math. 49, 157-266.

W. Robertson (1958), 'Completions of topological vector spaces', Proc. London Math. Soc. (3) 8, 242-257.

S. Saxon (1974a), 'Some normed barrelled spaces which are not Baire', Math. Ann. 209, 153-160.

S. Saxon (1974b), 'Two characterizations of linear Baire spaces', Proc. Amer. Math. Soc. 45, 204-208.

A. R. Todd and S. A. Saxon (1973), 'A property of locally convex Baire spaces', Math. Ann. 206, 23-34.

Division of Mathematics and Science

St John's University, Staten Island Campus

Staten Island, New York 10301

U.S.A. 